\title{
Can the NIST adsorption database be used to highlight potential materials for gas separation?
}

\author{
Paul lacomi $(\mathbb{D})^{\text {a, b, }}{ }^{*}$ and Philip L. Llewellyn (1D ${ }^{\text {a }}$ \\ ${ }^{\text {a }}$ Aix-Marseille University, CNRS, MADIREL UMR 7246, 13397 Marseille, France

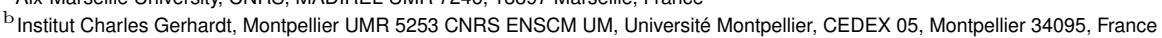 \\ *mail@pauliacomi.com
}

\begin{abstract}
Scientific literature is replete with descriptions of novel adsorbent materials, making the selection of such adsorbents for gas storage and separation a trudging task, and often resulting in overlooked materials. Here, we use a high throughput methodology to process a dataset of 28000 adsorption isotherms from the NIST adsorption database (ISODB) and generate key performance indicators applicable to ambient temperature binary separation on $\mathbf{1 5 0 0}$ materials in the collection, with $\mathbf{3 0}$ adsorbed guests. The procedure is validated against high-quality laboratory isotherms to confirm the accuracy of the derived indicators. The results are then collated in a powerful online dashboard, which can be used to explore the binary correlations. Finally, we use this toolchain to scrutinize several challenging and industrially relevant case studies and highlight some materials which may be promising for further analysis.
\end{abstract}

Keywords: gas separation ; high throughput processing ; data analysis

\section{Introduction}

A large proportion of the energy consumption of a raw materials plant goes into separating various components of the product stream. When the physical properties of the compounds to be separated are nearly identical, their concentration is low, or when complex mixtures are present, traditional processes which rely on phase change, like distillation, may be prohibitively expensive. Consequently, there are strong incentives to perfect and implement alternative separation avenues, such as adsorption or membrane based processes ${ }^{1}$.

In the case of gas separation, the potential use of porous materials may bring many-fold improvements in efficiency and selectivity. However, the selection process of a suitable material for a desired application is limited by the large possibility space of existing (or hypothetical) adsorbents. Zeolites, commonly employed as adsorbents, come in many real ${ }^{2}$ and possible ${ }^{3}$ structures. In the promising field of metal-organic frameworks (MOFs), more than 80000 structures have been experimentally identified in the Cambridge Structural Database (CSD) ${ }^{4,5}$ and many more predicted to exist ${ }^{6-8}$.

One desirable approach which has recently garnered much scientific interest is to predict well-performing materials through the use of computation. Such in-silico high throughput approaches usually focus on a "bottom-up" approach: to model or predict material properties and performance starting from their chemical structure, or descriptors thereof. Using such methods, possible materials have been identified for $\mathrm{CO}_{2}$ capture $^{9-12}$, Xe-Kr separation ${ }^{13,14}$ as well as storage of $\mathrm{CH}_{4},{ }^{5,15-17} \mathrm{H}_{2}{ }^{18-20}$, and $\mathrm{O}_{2}{ }^{21}$.

However, these methods cannot yet reliably generate predictions of synthesis pathways, thermal and chemical stability, or fully take into account structural features such as flexibility, defects, crystal size effects or interpenetration. While it may be only a matter of time until these challenges are surmounted (with promising advances being made ${ }^{22,23}$ ), there are likely many materials which already exist in the published literature

Electronic Supplementary Information (ESI) available: one PDF file with all referenced supporting information, and an animation of the online dashboard. but have not been considered for the specific function where they would excel. As a relevant example, a recent study has shown that one of the best currently performing thermoelectric materials could have been predicted 4 years before it was associated with this application ${ }^{24}$.

This raises the question whether identifying materials for a target separation could be feasible if it would be able to scan all available literature. Associating materials with single descriptive parameters, such as pore volume and surface area, has already been attempted ${ }^{25}$. However, the task of converting published adsorption data into a searchable database is not trivial, as isotherms are usually given as graphical figures rather than numerical data. Even if it would be possible to infer plot values, the isotherms are reported in a plethora of units, basis and formats. Excellent progress has been made by NIST $^{26}$ to compile such a database. Currently the NIST isotherm database (ISODB) comprises of more than 28000 isotherms recorded on over 4000 adsorbent materials, containing mostly MOFs but also zeolites, silicas, and carbons. While some of the datapoints are obtained from simulations, many of the isotherms in the ISODB originate from experiments, and are therefore a true indication of the performance of the real material.

The potential of exploring such a comprehensive dataset is clear. However, analysis should be performed carefully. Recently, Sholl and co-workers ${ }^{27}$ have published a report putting into question the reproducibility of isotherms, using $\mathrm{CO}_{2}$ adsorption data from the same NIST database. Their findings highlight a large variability inherent to measured isotherms, as on average "one in five $\mathrm{CO}_{2}$ isotherms [...] cannot be used to provide information that is qualitatively reliable about the properties of the material". A large contribution to this divergence is likely accounted for by the variation introduced through sample preparation and experimental methodology ${ }^{28}$. However, it is often the case that the most important factor in the repeatability of adsorption isotherms is not the measurement procedure, but the material itself.

From a pragmatic, applications standpoint, one can ask 
whether it is possible to use the database to identify materials of interest for gas separation. In the following work, we attempt to further investigate the ISODB dataset using high-throughput processing to generate and compare simple, one-dimensional key performance indicators (KPI), such as initial Henry constant, uptake and working capacity. A powerful interactive dashboard is constructed to explore the data as a function of various adsorbates, pressures and materials. Several well-known materials are then highlighted and compared with controlled laboratory experiments to validate our approach. We then attempt to draw conclusions from the dataset with respect to various common separations and a pressure swing adsorption (PSA) setup in mind.

\section{Materials and methods}

2.1. Isotherm selection and conversion. The entire dataset in the NIST adsorption database was downloaded using the publicly available API, which yielded $\approx 28000$ single component isotherms. All further processing of the database was performed using the pyGAPS software which was previously developed by us $^{29}$. Data in the ISODB comes from a variety of published sources, including experimental and simulated isotherms with a variety of adsorbates, temperatures and units. In order to narrow down the dataset and ensure comparability the following steps were taken.

- Only isotherms measured with 38 common adsorbates were selected, such as simple probes, light hydrocarbons and common vapours.

- Isotherms which could not be converted to $\mathrm{mmolg}^{-1}$ vs. bar were discarded. This includes data reported on a volumetric material basis, simulation data which is reported per unit cell, and fractional coverage isotherms. All remaining isotherms were then converted.

- No isotherms with less than 6 measurement points were selected, to ensure enough datapoints for latter processing.

- Possible outliers were removed by selecting isotherms with pressure points under 100 bar, maximum loading under $100 \mathrm{mmol} \mathrm{g}^{-1}$ and an isotherm temperature of under $443 \mathrm{~K}$. Any such isotherms are likely errors in the data collection process and have little to no physical meaning.

The process of data collation reduced the number of isotherms to $\approx 15800$. A distribution of the isotherms as a function of adsorbate and temperature can be found in Fig. 1. Isotherms were then selected in a (303 \pm 10$) \mathrm{K}$ temperature range. The resulting dataset contains 5800 isotherms on 1503 materials with 30 adsorbates. It was assumed that this range of temperature does not have a stark effect on the amount adsorbed or other isotherm features. It should be noted that certain temperature-critical effects, such as structural transitions, may be sensitive to this approach.

2.2. KPI selection. Predicting the separation performance of a material starting from pure component isotherms is not trivial. Most attempts at creating an universal application performance indicator (API) for a PSA separation rely on a combination of scalar properties such as: total uptake, working capacity, selectivity (approximated or predicted by methods such as Ideal Adsorbed Solution Theory), enthalpy of adsorption, surface area, etc. ${ }^{30}$. The performance indicator is often further tuned with specific factors to account for the process in question, desired separation efficiency, regeneration cost and other constraints.

When working with a variable dataset, overprocessing of the data will propagate errors to an unmanageable degree. We therefore selected KPI which can be calculated directly from a pure component isotherm and are relatively robust. We have chosen three such parameters: initial Henry's constant $K_{H, i}$, loading at a single pressure $L(p)$, and working capacity between two pressures $W C\left(p_{1}, p_{2}\right)$.

The initial Henry constant can serve as a useful metric to evaluate the strength of the interaction between a probe and the adsorption site. What is more, the ratio of two Henry constants can be a useful shorthand for estimating the selectivity of a material ${ }^{31}$. Two possible methods of obtaining the initial Henry constant were considered: linear and virial model fitting. The linear method fits a line to the initial part of the isotherm, more sensitive to the quality of the low pressure data, but applicable to all isotherm types and features, The virial model attempts to fit the entire dataset with a virial isotherm ${ }^{32}$, then evaluates the resulting function at zero pressure. Full details of the implementation of these methods are available elsewhere $^{29}$. It was found that both methods produce similar results, as seen in Fig. S1. The slope method was finally selected, as it is generally applicable. Uptake was calculated by using a first order spline to interpolate between isotherm points at various set pressures. No extrapolation above the maximum recorded pressure point was performed. Working capacity is calculated as the difference of two uptakes at different pressures. These latter KPI can be used as performance metrics when considering the quantity of material required for a separation. Furthermore, for a given pair of gases, the ratio of uptakes can also be a means to highlight materials of potential interest. Including the differential enthalpy of adsorption as a KPI, as proposed by Wiersum et al. ${ }^{30}$, would be desirable. However, the isotherms contained in the ISODB do not contain direct enthalpy measurements, and the use of the Clausius-Clapeyron method is susceptible to error. Therefore, no inclusion of this metric was possible.

2.3. Data consolidation and outlier detection. The KPI from the previous section were calculated for each isotherm selected. Consolidating the data in order to obtain a single representative point for each material was required. Therefore, for each unique adsorbent the resulting KPI values were first passed through gross outlier rejection, in order to remove any spurious values resulting from non-physical isotherms. As such values outside the $1 e \pm 8$ range were removed from further calculations. For robust outlier detection, the interquartile range (IQR) for distributions which had more than 4 datapoints was first calculated. Points were considered outliers if they were 1.5 IQR below or above the lower or upper quartile, respectively. For datasets with less than 4 values, no points were considered as outliers. The median of the remaining points was taken as the final KPI value. To give an indication of scale, the standard deviation was used. In the case of materials with a single isotherm, the values were calculated directly.

All the processing steps and resulting datasets are available to download as Jupyter notebooks and JSON files on the authors' GitHub page at https://github.com/pauliacomi/ separation-explorer. 


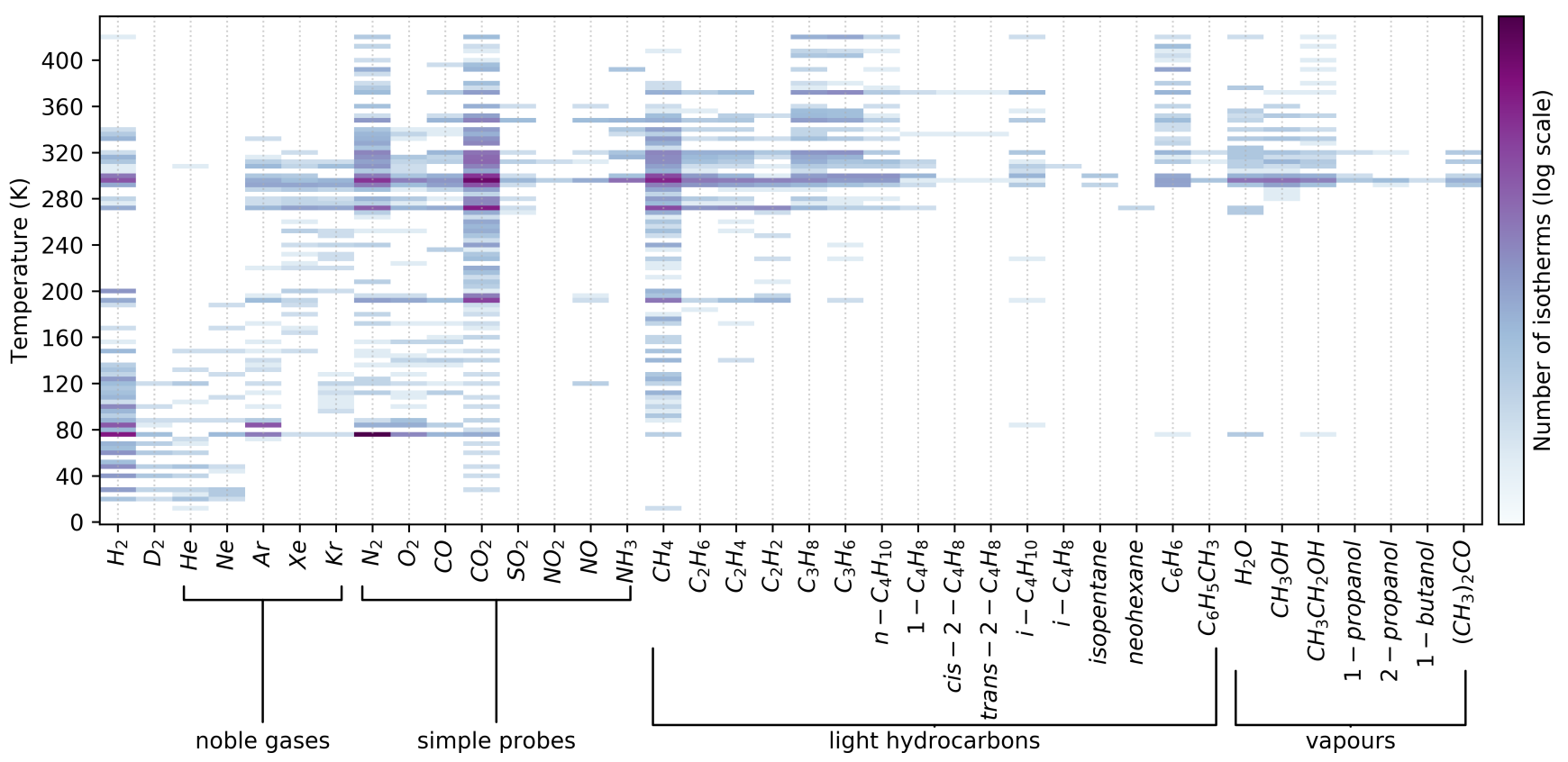

Fig. 1. A graphical description of the NIST ISODB dataset: the selected 15800 isotherms presented per adsorbate used and temperature at which the measurement took place. Colour represents number of isotherms on a log scale.

\section{Results and discussion}

3.1. Isotherm reliability. As it was clearly shown in the report by Sholl and co-workers ${ }^{27}$, inherent variability exists in published adsorption data. This reproducibility issue can also be seen in an analysis of specific surface area as calculated from nitrogen physisorption at $77 \mathrm{~K}$ (available in the SI). Therefore, for the employed processing methods to be trusted, the statistical approach detailed in the methods section should first be validated. For this purpose, we have used an internal isotherm database from the MADIREL lab, which contains collated results from our previous work and can be considered a high-quality reference. Most of these isotherms come from the work of Wiersum et al. ${ }^{30}$. Our database consists of $\approx 450$ isotherms measured on prototypical materials with various simple probe gases and light hydrocarbons.

First, the databases were cross-referenced to find measurements with the same probes on materials present in both datasets. Then, the same KPI calculation methods and derivation of single-point values were applied to the MADIRELderived isotherms as to the NIST dataset. The common datapoints are displayed in Fig. 2.

It can be seen that the two KPI correspond surprisingly well. An obvious spurious point appears in butane adsorption on $\mathrm{CuBTC}$, but otherwise a good correlation is seen between the two databases. While this validation does not test all materials, it gives a good indication that the statistical methods we have applied can be considered adequate for most cases.

3.2. Dashboard. Exploring such a large dataset is difficult with conventional methods. We have therefore constructed an interactive dashboard, which can be used to sift through the resulting relationships. The dashboard is accessible online at https://separation-explorer.herokuapp.com and can also be downloaded and executed locally from the previous GitHub

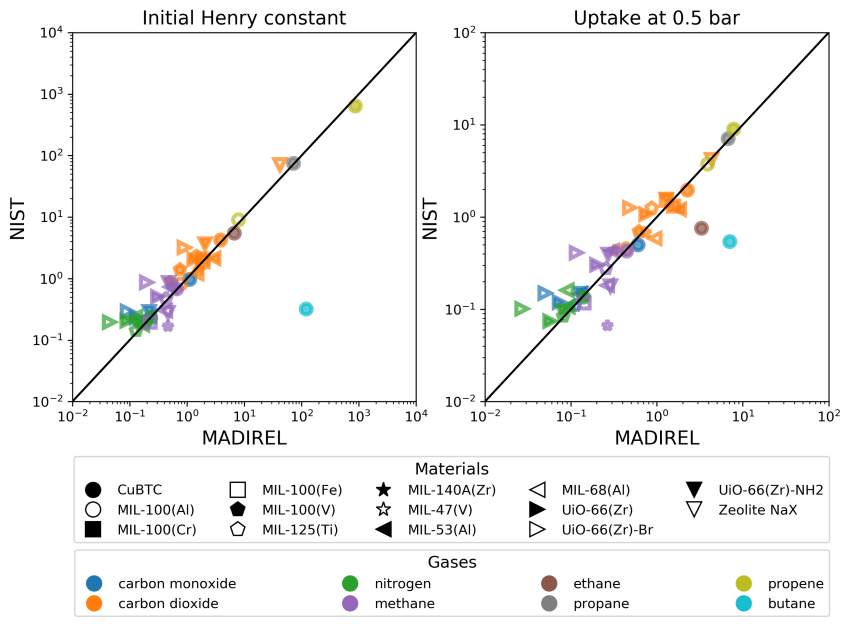

Fig. 2. Comparison between KPI calculated on the basis of the internal MADIREL dataset and the materials from the NIST database. Colours denote gases, while marker shape denotes material. Error margins are not displayed.

link. To operate, the user selects two probes from the list of available adsorbates. The dashboard then creates a table of all corresponding materials, as well as graphs of the previously discussed KPI. A snapshot of the $\mathrm{CH}_{4}$ and $\mathrm{CO}_{2}$ correlation is presented in Fig. 3. Each point represents the median KPI value for one material. Points only appear when both KPI are available for each gas selected. The colour of the points is used to indicate the number of datapoints it represents, with grey points signifying values calculated from a single isotherm on each material.

The dashboard allows for a high level of interactivity. Hovering over a point will display the material name, as well as details about its dataset. One or multiple points can be 
Gas 1

methane

\begin{tabular}{r|l|l|l|}
\hline$\square$ & Material & KH2/KH1 & PSA-API \\
\hline$\square$. & MIL-53 & $14.60 \mathrm{k}$ & $\mathrm{NaN}$ \\
\hline$\square$. & Zeolite LS-Ca-KFI & 744.08 & 880.92 \\
\hline$\square$. & TPA-Activated at 90C & 498.16 & $\mathrm{NaN}$ \\
\hline$\square$. & MOF-1M'-Li & 440.33 & 934.24 \\
\hline$\square$. & C10H5MnN3O4 & 434.90 & $\mathrm{NaN}$ \\
\hline$\square$. & Zeolite LS-Sr-KFI & 402.51 & 587.62 \\
\hline$\square$. & activated \{[Zn4(bpydb)3(datz)2(H2O)](DMF)4(Et & 332.08 & $\mathrm{NaN}$ \\
\hline$\square$. & Zeolite NaX & 251.60 & 129.42 \\
\hline$\square$. & Mg-MOF-74 (3) & 197.88 & $\mathrm{NaN}$ \\
\hline$\square$. & Chabazite & 192.67 & $\mathrm{NaN}$ \\
\hline$\square$. & TBA-Activated at 180C & 162.17 & $\mathrm{NaN}$ \\
\hline$\square$. & Zeolite Na-A & 157.86 & $\mathrm{NaN}$ \\
\hline$\square$. & TPA-Activated at 180C & 149.12 & $\mathrm{NaN}$ \\
\hline$\square$. & COF-6 & 140.29 & 172.94 \\
\hline$\square$. & Mg2(dobdc) & 133.27 & $\mathrm{NaN}$ \\
\hline & & &
\end{tabular}

Uptake at selected pressure

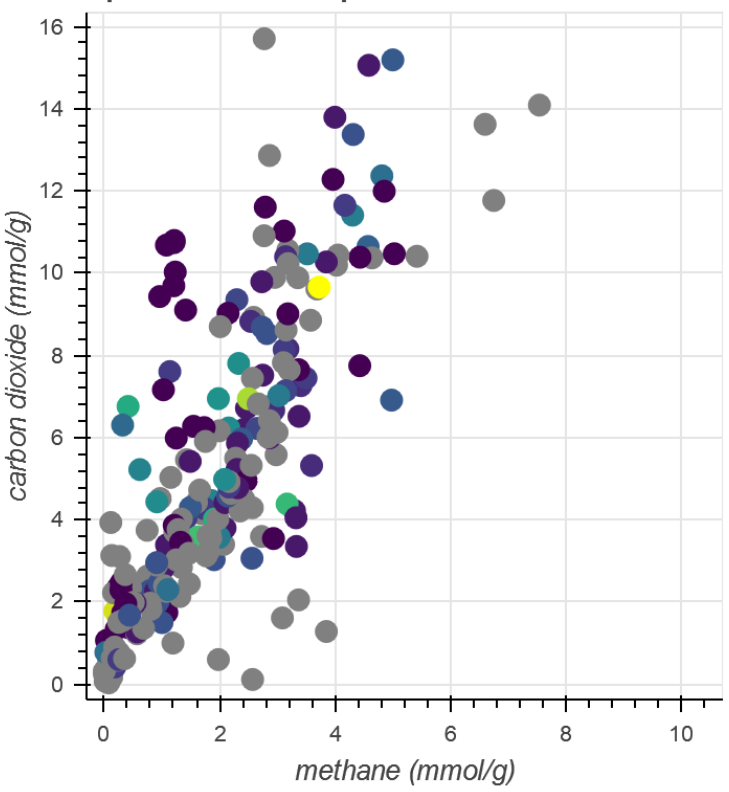

Pressure: 7
Gas 2

carbon dioxide

生

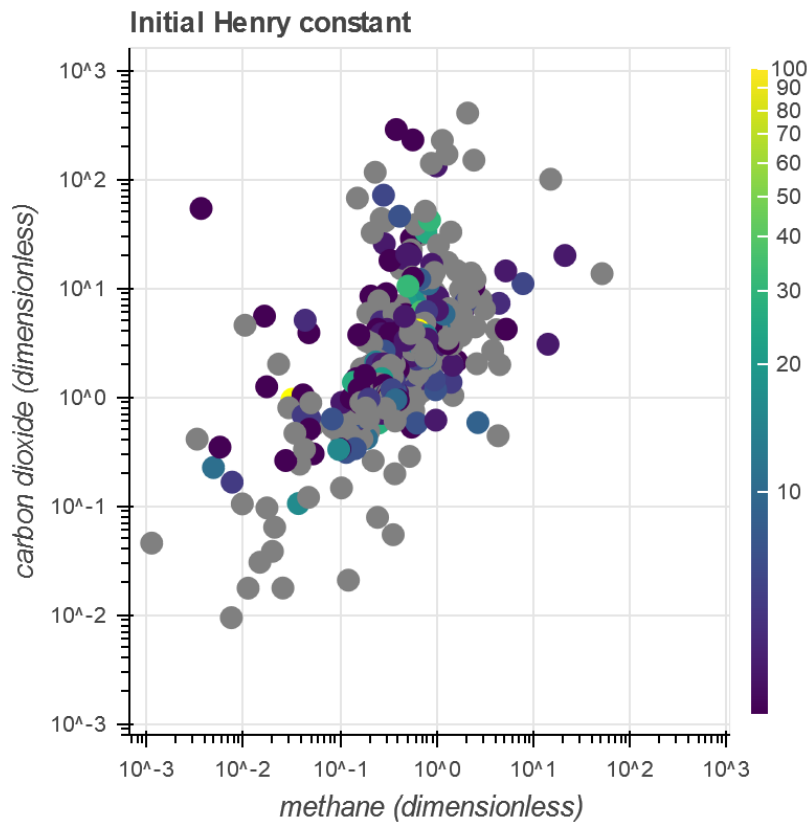

Working capacity in selected range

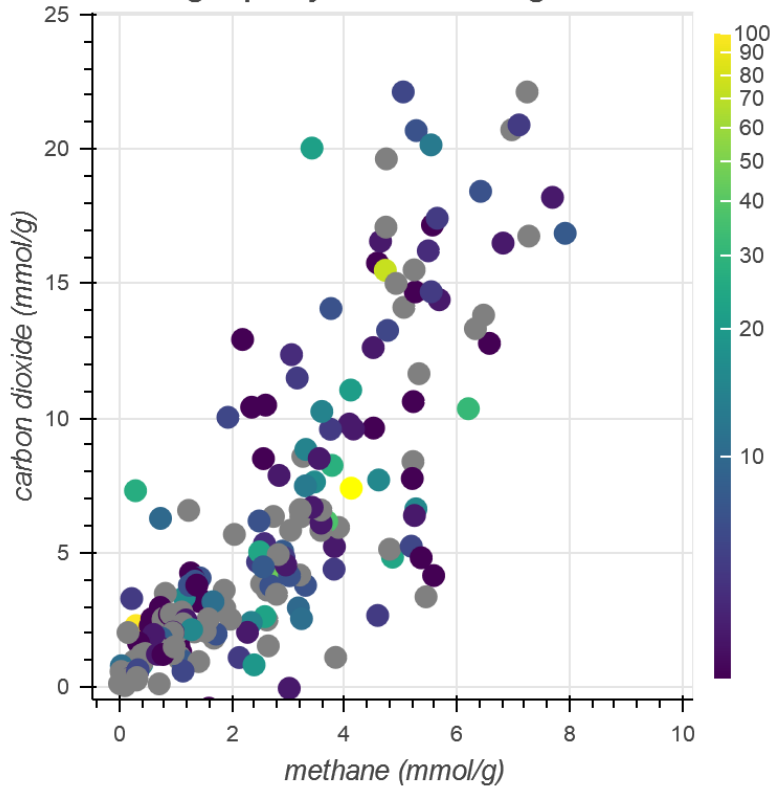

Working capacity: 1 .. 15

Fig. 3. Separation explorer dashboard overview of the $\mathrm{CO}_{2} / \mathrm{CH}_{4}$ dataset. Top left: the table of all materials with at least one point on display, sorted by ratio of Henry constants Top right: Henry constant correlation. Bottom left: uptake correlation at 10 bar pressure, value user-selectable in the slider beneath. Bottom right: working capacity in the 1 bar to 15 bar pressure range, similarly selectable. Colour of the points is representative of the number of isotherms available per material, with grey denoting only one datapoint for each gas. The NaN points are a result of no data being available for the material in the working pressure range. 
selected directly from the table or the graphs in order to display the confidence range of the KPI as well as the material name. The hovered or selected material is highlighted in all relationships, to allow for an overview of its performance.

The pressure at which uptake is calculated, as well as the pressure range in which working capacity is determined is userselectable through the sliders under the respective graphs. The KPI at the selected pressure are displayed on-the-fly which allows to track their evolution at various increments. An example of such an animation can be seen in Supplementary Video 1.

If one material is selected, the isotherms which were used to calculate the respective KPI for each adsorbate are displayed below the dashboard, together with the median calculated uptake. The user can click on an isotherm to follow the link to the respective ISODB page of the publication.

Besides displaying the results in a graphical form, two performance indicators geared towards separation are calculated for the selected gases and displayed in the table next to each material. The simplest indicator is the ratio of the two Henry constants, as an estimation for the selectivity of the material towards the two gases as proposed by Knaebel ${ }^{31}$. A PSA selection parameter is also calculated, similar to the one described by Rege and Yang ${ }^{33}$, but using the Henry constant ratio in place of the selectivity.

$$
S_{P S A}=\frac{W C_{1}}{W C_{2}} \alpha_{12} \approx \frac{W C_{1}}{W C_{2}} \frac{K_{H, 1}}{K_{H, 2}}
$$

The list can be sorted in order to highlight adsorbents which may be well suited for a separation according to these indicators.

3.3. Case studies for varius separations. After validating the processing methodology and developing a toolset to explore the database from a binary separation perspective, we now turn to several case studies to exemplify the insights that can be extracted. We also attempt to highlight materials with known separation performance, as well as some less explored alternatives.

3.3.1. $\mathbf{C O}_{2} / \mathbf{C H}_{\mathbf{4}}$. Observing the graphs displayed in Fig. 3, we can discern several self-evident trends. The adsorption of $\mathrm{CO}_{2}$ clearly takes precedence over that of $\mathrm{CH}_{4}$ in nearly all materials. This is to be expected, due to the quadrupole moment of former molecule and the relatively non-specific dispersion interactions of the latter.

The top 10 best performing materials as ranked by Henry constant ratio and the PSA selection parameter calculated between 1 and 5 bar are displayed in Fig. 4. Unsurprisingly, many zeolites, such as Zeolite LS-Ca-KFI or Zeolite NaX are highlighted as top performers when considering the ratio of Henry constants. It is well known that such materials are promising for carbon capture from natural gas ${ }^{9}$ due to their strong interaction with $\mathrm{CO}_{2}$. When considering the PSA selection parameter, which also takes into account working capacity, zeolites are less desirable due to the small uptake difference between ambient pressure and higher pressures. Here, several amine-functionalised mesoporous MCM-41 materials emerge as possible candidates due to their high capacity for $\mathrm{CO}_{2}$ adsorption compared to $\mathrm{N}_{2}$.

In both graphs, the MIL-53 family of materials is seen to perform very well, which can be traced to to their flexible
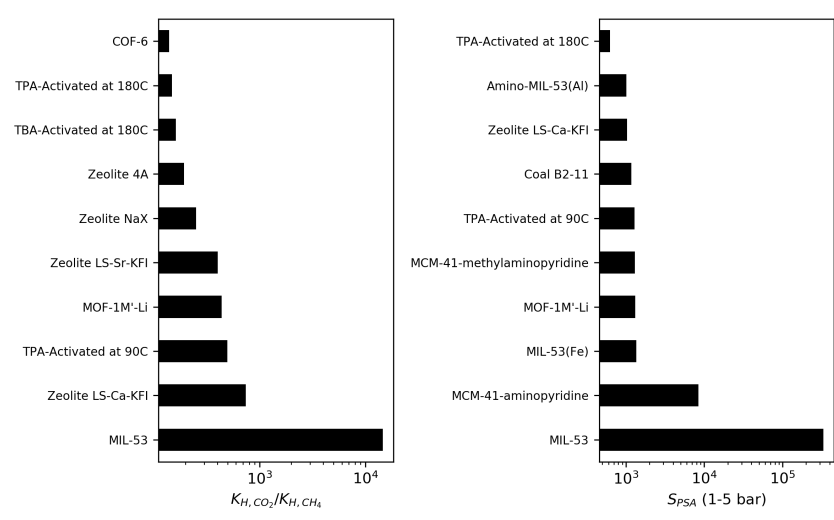

Fig. 4. The top 10 materials for $\mathrm{CO}_{2}-\mathrm{CH}_{4}$ separations as ranked by the ratio of Henry constants (left) and the PSA selection parameter (right). Material labels are taken directly form the ISODB.

gate-opening behaviour. For example, in the iron variant MIL$53(\mathrm{Fe})^{34}$, the $\mathrm{CO}_{2}$ opening step occurs at 5 bar while $\mathrm{CH}_{4}$ does not re-open the structure at this pressure. It should be noted that the top performing material designated as "MIL-53" in Fig. 4 is actually the amino-terephtalic acid linker variant, or $\mathrm{MIL}-53(\mathrm{Al})-\mathrm{NH}_{3}{ }^{35}$.

One further material which appears to show good performance according to both predictors is Sr-UPRM-5, a flexible titanium silicate templated either with tetrapropylammonium (TPA) or tetrabutylammonium (TBA), originally reported by Marcano-González et al. ${ }^{36}$ and herein referred to as TPAActivated or TBA-Activated, respectively.

3.3.2. $\mathbf{C}_{3} \mathbf{H}_{6} / \mathbf{C}_{3} \mathbf{H}_{8}$. The difficulty of thermodynamic separation of a propane/propylene mixture can be discerned from the KPI correlation observed in Fig. 5. Both the Henry constant and the uptake at 0.5 bar are fairly closely matched.
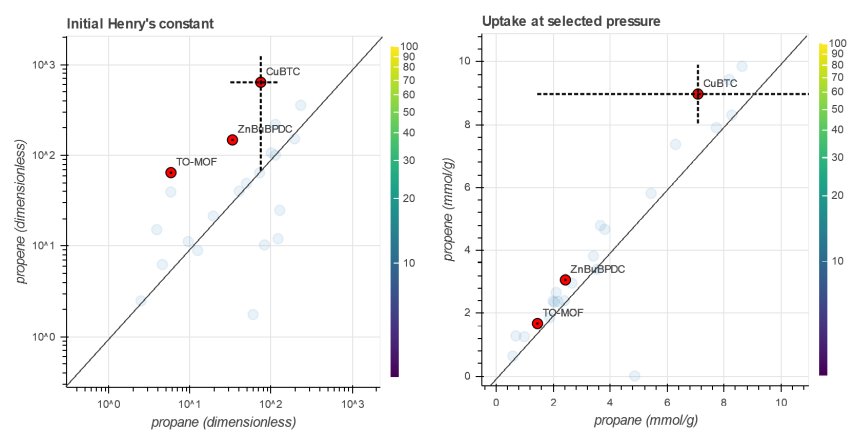

Fig. 5. Correlation of Henry constant (left) and uptake at 0.5 bar (right) for propane and propylene, with several highlighted materials. Lines are guide for the eye at $x=y$.

Here, we can highlight three materials which are selective towards propylene: CuBTC, TO-MOF and ZnBuPBDC. It is well known that CuBTC preferentially adsorbs the unsaturated component due to the strong interaction of its copper paddlewheel with the double bond ${ }^{37}$. The second material is part of an isostructural series of pillared MOFs tuned for kinetic separations of propylene/propane mixtures ${ }^{38}$. However, it is worth pointing out that, to our knowledge, no study of the separation performance of $\mathrm{ZnBuPBDC}$ was ever performed ${ }^{39}$. 
Another observation can be made by noting that an inverse selectivity can be seen in ZIF-7, where propane is preferentially adsorbed over propylene ${ }^{40}$, predicted by its location in the lower right quadrant of the Henry constant correlation. It should also be specified that the calculated KPI take into account only equilibrium isotherms. Therefore, any kinetic effects cannot be assessed, and are indeed more likely to lead to well-performing materials.

3.3.3. Contaminants in post-combustion carbon capture. Traces of water or sulphur and nitrogen oxides $\left(\mathrm{SO}_{\mathrm{X}} / \mathrm{NO}_{\mathrm{X}}\right)$ in flue gas have a profound impact on post-combustion carbon capture. Due to strong interactions with the adsorbent material and poor regenerability, such contaminants are detrimental to $\mathrm{CO}_{2}$ adsorption performance. Indeed, this can be visualised by comparing the KPI for the desired adsorbed component and such contaminants. If selecting $\mathrm{H}_{2} \mathrm{O} / \mathrm{CO}_{2}$ we can observe that the initial Henry constant ratio is above unity for $80 \%$ of materials. This suggests a preference for water adsorption over carbon dioxide. When the two components are $\mathrm{SO}_{2} / \mathrm{CO}_{2}$, there are no ratios below 2 .
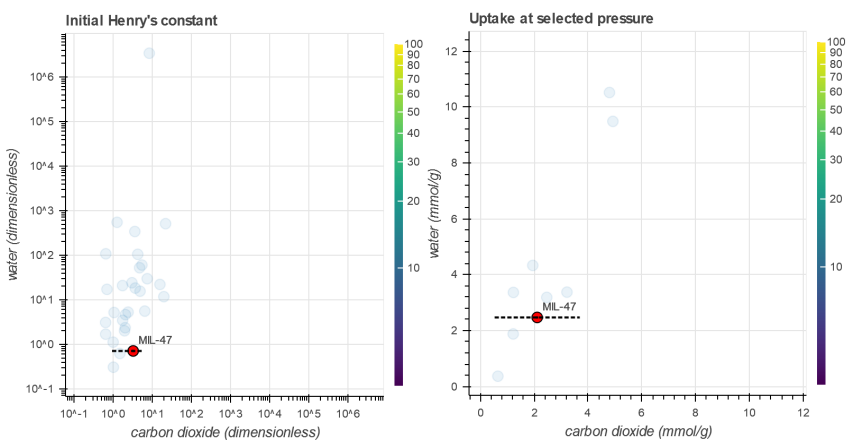

Fig. 6. Correlation of Henry constant (left) and uptake at 1 bar (right) for $\mathrm{H}_{2} \mathrm{O}$ and $\mathrm{CO}_{2}$, with MIL-47(V) highlighted. Most materials are seen to have a preference for water.

If considering materials which have a low selectivity for $\mathrm{CO}_{2}$, it may be possible to identify adsorbents which could be well suited for impurity-rich inlet streams. Here, we can highlight MIL-47(V), ${ }^{41}$ as a MOF which appears to show a weaker affinity towards water. It should be noted that the only water isotherm available originates from a simulation study $^{42}$

\section{Conclusions}

In this work we have shown that processing the NIST dataset can allow for significant insight into the performance of published materials for specific separations. By comparing the ISODB dataset against an internal high-quality standard, we prove that the variability present in publication data does not broadly affect the calculation of KPI such as Henry constant, uptake or working capacity. The obtained indicators were then incorporated in an online dashboard, which allowed visual exploration of the material performance and trends of various applications.

It should be noted that this study can only highlight a narrow range of existing materials for cherry-picked applications, with undoubtedly many more which may be of interest for different topics. We encourage the reader to explore the online dashboard at their own convenience.
As the methods of text scraping and digitisation improve, the amount of experimentally-derived data available for further processing will only increase. With a more powerful statistical approach or a selected subset of isotherms like the internal MADIREL database, more complex processing may be performed, such as prediction of binary separation selectivity through Ideal Adsorbed Solution Theory. In the future, we can also envisage a combined approach that would use such real-world dataset processing to validate the in-silico high throughput simulation methods currently employed.

\section{Acknowledgements}

The authors would like to thank Dan Siderius from NIST for his advice and extensive discussions. PI is thankful to Rocio Semino for the insightful conversations. We are grateful to Andrew Wiersum and the other past and present members of the MADIREL lab for recording the high quality data the internal database is based on, and to our collaborators which supplied the adsorbent samples. This work has received funding from the European Union's Horizon 2020 research and innovation programme under the Marie Sklodowska-Curie grant agreement No 641887 (project acronym: DEFNET).

\section{References}

1. Sholl, D.S. and Lively, R.P. "Seven chemical separations to change the world." Nature, 2016 532(7600):435-437. doi:10.1038/532435a

2. Baerlocher, C.; McCusker, L.B.; Olson, D.; Meier, W.M.; International Zeolite Association; and Structure Commission. Atlas of Zeolite Framework Types. Published on behalf of the Structure Commission of the International Zeolite Association by Elsevier, Amsterdam; Boston, 2007. ISBN 978-0-08-055434-1 978-0-444-53064-6. OCLC: 182747982

3. Foster, M. and Treacy, M. "A Database of Hypothetical Zeolite Structures." http://www.hypotheticalzeolites.net, ????

4. Moghadam, P.Z.; Li, A.; Wiggin, S.B.; Tao, A.; Maloney, A.G.P.; Wood, P.A.; Ward, S.C.; and Fairen-Jimenez, D. "Development of a Cambridge Structural Database Subset: A Collection of Metal-Organic Frameworks for Past, Present, and Future." Chemistry of Materials, 2017. 29(7):2618-2625. doi:10.1021/acs.chemmater.7b00441

5. Chung, Y.G.; Camp, J.; Haranczyk, M.; Sikora, B.J.; Bury, W.; Krungleviciute, V.; Yildirim, T. Farha, O.K.; Sholl, D.S.; and Snurr, R.Q. "Computation-Ready, Experimental Metal-Organic Frameworks: A Tool To Enable High-Throughput Screening of Nanoporous Crystals." Chemistry of Materials, 2014. 26(21):6185-6192. doi:10.1021/cm502594j

6. Wilmer, C.E.; Leaf, M.; Lee, C.Y.; Farha, O.K.; Hauser, B.G.; Hupp, J.T.; and Snurr, R.Q. "Large-scale screening of hypothetical metal-organic frameworks." Nature Chemistry, 2012. 4(2):83-89. doi:10.1038/nchem.1192

7. Bureekaew, S. and Schmid, R. "Hypothetical 3D-periodic covalent organic frameworks: Exploring the possibilities by a first principles derived force field." CrystEngComm, 2013. 15(8):1551. doi:10.1039/c2ce26473k

8. Baburin, I.A.; Leoni, S.; and Seifert, G. "Enumeration of Not-Yet-Synthesized Zeolitic Zinc Imidazolate MOF Networks: A Topological and DFT Approach." The Journal of Physical Chemistry B, 2008. 112(31):9437-9443. doi:10.1021/jp801681w

9. Braun, E.; Zurhelle, A.F.; Thijssen, W.; Schnell, S.K.; Lin, L.C.; Kim, J.; Thompson, J.A.; and Smit, B. "High-throughput computational screening of nanoporous adsorbents for $\mathrm{CO}_{2}$ capture from natural gas." Molecular Systems Design \& Engineering, 2016. 1(2):175-188. doi:10.1039/C6ME00043F

10. Chung, Y.G.; Gómez-Gualdrón, D.A.; Li, P.; Leperi, K.T.; Deria, P.; Zhang, H.; Vermeulen, N.A.; Stoddart, J.F.; You, F.; Hupp, J.T.; Farha, O.K.; and Snurr, R.Q. "In silico discovery of metal-organic frameworks for precombustion $\mathrm{CO}_{2}$ capture using a genetic algorithm." Science Advances, 2016. 2(10):e1600909. doi:10.1126/sciadv.1600909

11. Li, S.; Chung, Y.G.; Simon, C.M.; and Snurr, R.Q. "High-Throughput Computational Screening of Multivariate Metal-Organic Frameworks (MTV-MOFs) for $\mathrm{CO}_{2}$ Capture." The Journal of Physical Chemistry Letters, 2017. 8(24):6135-6141. doi:10.1021/acs.jpclett.7b02700

12. Park, J.; Lively, R.P.; and Sholl, D.S. "Establishing upper bounds on $\mathrm{CO}_{2}$ swing capacity in sub-ambient pressure swing adsorption via molecular simulation of metal-organic frameworks." Journal of Materials Chemistry A, 2017. 5(24):12258-12265. doi:10.1039/C7TA02916K

13. Simon, C.M.; Mercado, R.; Schnell, S.K.; Smit, B.; and Haranczyk, M. "What Are the Best Materials To Separate a Xenon/Krypton Mixture?" Chemistry of Materials, 2015. 27(12):44594475. doi:10.1021/acs.chemmater.5b01475

14. Banerjee, D.; Simon, C.M.; Plonka, A.M.; Motkuri, R.K.; Liu, J.; Chen, X.; Smit, B.; Parise, J.B.; Haranczyk, M.; and Thallapally, P.K. "Metal-organic framework with optimally selective xenon adsorption and separation." Nature Communications, 2016. 7(1):ncomms11831. doi:10.1038/ncomms 11831

15. Simon, C.M.; Kim, J.; Lin, L.C.; Martin, R.L.; Haranczyk, M.; and Smit, B. "Optimizing nanoporous materials for gas storage." Physical Chemistry Chemical Physics, 2014. 16(12):5499. doi:10.1039/c3cp55039g 
16. Pardakhti, M.; Moharreri, E.; Wanik, D.; Suib, S.L.; and Srivastava, R. "Machine Learning Using Combined Structural and Chemical Descriptors for Prediction of Methane Adsorption Performance of Metal Organic Frameworks (MOFs)." ACS Combinatorial Science, 2017. 19(10):640-645. doi:10.1021/acscombsci.7b00056

17. Lee, Y.; Barthel, S.D.; Dłotko, P.; Moosavi, S.M.; Hess, K.; and Smit, B. "High-Throughput Screening Approach for Nanoporous Materials Genome Using Topological Data Analysis: Application to Zeolites." Journal of Chemical Theory and Computation, 2018. 14(8):44274437. doi:10.1021/acs.jctc.8b00253

18. Gómez-Gualdrón, D.A.; Colón, Y.J.; Zhang, X.; Wang, T.C.; Chen, Y.S.; Hupp, J.T.; Yildirim, T.; Farha, O.K.; Zhang, J.; and Snurr, R.Q. "Evaluating topologically diverse metal-organic frameworks for cryo-adsorbed hydrogen storage." Energy \& Environmental Science, 2016. 9(10):3279-3289. doi:10.1039/C6EE02104B

19. Thornton, A.W.; Simon, C.M.; Kim, J.; Kwon, O.; Deeg, K.S.; Konstas, K.; Pas, S.J.; Hill, M.R.; Winkler, D.A.; Haranczyk, M.; and Smit, B. "Materials Genome in Action: Identifying the Performance Limits of Physical Hydrogen Storage." Chemistry of Materials, 2017. 29(7):2844-2854. doi:10.1021/acs.chemmater.6b04933

20. Bucior, B.J.; Bobbitt, N.S.; Islamoglu, T.; Goswami, S.; Gopalan, A.; Yildirim, T.; Farha, O.K.; Bagheri, N.; and Snurr, R.Q. "Energy-based descriptors to rapidly predict hydrogen storage in metal-organic frameworks." Molecular Systems Design \& Engineering, 2019. 4(1):162-174. doi:10.1039/C8ME00050F

21. Moghadam, P.Z.; Islamoglu, T.; Goswami, S.; Exley, J.; Fantham, M.; Kaminski, C.F.; Snurr, R.Q.; Farha, O.K.; and Fairen-Jimenez, D. "Computer-aided discovery of a metal-organic framework with superior oxygen uptake." Nature Communications, $2018 . \quad$ 9(1):1378. doi:10.1038/s41467-018-03892-8

22. Moghadam, P.Z.; Rogge, S.M.; Li, A.; Chow, C.M.; Wieme, J.; Moharrami, N.; AragonesAnglada, M.; Conduit, G.; Gomez-Gualdron, D.A.; Van Speybroeck, V.; and Fairen-Jimenez, D. "Structure-Mechanical Stability Relations of Metal-Organic Frameworks via Machine Learning." Matter, 2019. p. S2590238519300062. doi:10.1016/j.matt.2019.03.002

23. Evans, J.D. and Coudert, F.X. "Predicting the Mechanical Properties of Zeolite Frameworks by Machine Learning." Chemistry of Materials, 2017. 29(18):acs.chemmater.7b02532. doi:10.1021/acs.chemmater.7b02532

24. Tshitoyan, V.; Dagdelen, J.; Weston, L.; Dunn, A.; Rong, Z.; Kononova, O.; Persson, K.A.; Ceder, G.; and Jain, A. "Unsupervised word embeddings capture latent knowledge from materials science literature." Nature, 2019. 571(7763):95-98. doi:10.1038/s41586-019-13358

25. Park, S.; Kim, B.; Choi, S.; Boyd, P.G.; Smit, B.; and Kim, J. "Text Mining Metal-Organic Framework Papers." Journal of Chemical Information and Modeling, 2018. 58(2):244-251. doi:10.1021/acs.jcim.7b00608

26. Siderius, D.; Shen, V.; and Johnson, R. "NIST / ARPA-E Database of Novel and Emerging Adsorbent Materials." 2015. doi:10.18434/T43882

27. Park, J.; Howe, J.D.; and Sholl, D.S. "How Reproducible Are Isotherm Measurements in Metal-Organic Frameworks?" Chemistry of Materials, 2017. 29(24):10487-10495. doi:10.1021/acs.chemmater.7b04287

28. Nguyen, H.G.T.; Espinal, L.; van Zee, R.D.; Thommes, M.; Toman, B.; Hudson, M.S.L.; Mangano, E.; Brandani, S.; Broom, D.P.; Benham, M.J.; Cychosz, K.; Bertier, P.; Yang, F.; Krooss, B.M.; Siegelman, R.L.; Hakuman, M.; Nakai, K.; Ebner, A.D.; Erden, L.; Ritter, J.A.; Moran, A.; Talu, O.; Huang, Y.; Walton, K.S.; Billemont, P.; and De Weireld, G. "A reference high-pressure $\mathrm{CO} 2$ adsorption isotherm for ammonium ZSM-5 zeolite: Results of an interlaboratory study." Adsorption, 2018. doi:10.1007/s10450-018-9958-x

29. Paul lacomi and Philip L. Llewellyn. "pyGAPS: A Python-Based Framework for Adsorption Isotherm Processing and Material Characterisation." 2019. doi:10.26434/chemrxiv.7970402.v1

30. Wiersum, A.D.; Chang, J.S.; Serre, C.; and Llewellyn, P.L. "An Adsorbent Performance Indicator as a First Step Evaluation of Novel Sorbents for Gas Separations: Application to Metal-Organic Frameworks." Langmuir, 2013. 29(10):3301-3309. doi:10.1021/la3044329

31. Knaebel KS. "For Your Next Separation Consider Adsorption (Vol 102, Pg 92, 1995)." Chemical Engineering, 1995. 102(12):8-8

32. Myers, A.L. "Thermodynamics of adsorption in porous materials." AlChE Journal, 2002. 48(1):145-160. doi:10.1002/aic.690480115

33. Rege, S.U. and Yang, R.T. "A simple parameter for selecting an adsorbent for gas separation by pressure swing adsorption." Separation science and technology, 2001. 36(15):3355-3365

34. Llewellyn, P.L.; Horcajada, P.; Maurin, G.; Devic, T.; Rosenbach, N.; Bourrelly, S.; Serre, C.; Vincent, D.; Loera-Serna, S.; Filinchuk, Y.; and Férey, G. "Complex Adsorption of Short Linear Alkanes in the Flexible Metal-Organic-Framework MIL-53(Fe)." Journal of the American Chemical Society, 2009. 131(36):13002-13008. doi:10.1021/ja902740r

35. Couck, S.; Denayer, J.F.M.; Baron, G.V.; Rémy, T.; Gascon, J.; and Kapteijn, F. "An Amine-Functionalized MIL-53 Metal-Organic Framework with Large Separation Power for $\mathrm{CO}_{2}$ and $\mathrm{CH}_{4}$." Journal of the American Chemical Society, 2009. 131(18):6326-6327. doi:10.1021/ja900555r

36. Marcano-González, M.E.; Fu, R.; and Hernández-Maldonado, A.J. "Long- and Local-Range Structural Changes in Flexible Titanium Silicates with Variable Faulting upon Thermal Treatment and Corresponding Adsorption and Particle Size Polydispersity-Corrected Diffusion Parameters for $\mathrm{CO}_{2} / \mathrm{CH}_{4}$ Separation." Industrial \& Engineering Chemistry Research, 2015. 54(1):207-216. doi:10.1021/ie5046044

37. Rubeš, M.; Wiersum, A.D.; Llewellyn, P.L.; Grajciar, L.; Bludský, O.; Nachtigall, P.; Rubes, M.; Wiersum, A.D.; Llewellyn, P.L.; Grajciar, L.; Bludský, O.; and Nachtigall, P. "Adsorption of propane and propylene on CuBTC metal-organic framework: Combined theoretical and experimental investigation." Journal of Physical Chemistry C, 2013. 117(21):11159-11167. doi:10.1021/jp401600v

38. Lee, C.Y.; Bae, Y.S.; Jeong, N.C.; Farha, O.K.; Sarjeant, A.A.; Stern, C.L.; Nickias, P.; Snurr, R.Q.; Hupp, J.T.; and Nguyen, S.T. "Kinetic Separation of Propene and Propane in MetalOrganic Frameworks: Controlling Diffusion Rates in Plate-Shaped Crystals via Tuning of Pore Apertures and Crystallite Aspect Ratios." Journal of the American Chemical Society, 2011. 133(14):5228-5231. doi:10.1021/ja200553m
39. Keceli, E.; Hemgesberg, M.; Grünker, R.; Bon, V.; Wilhelm, C.; Philippi, T.; Schoch, R.; Sun Y.; Bauer, M.; Ernst, S.; Kaskel, S.; and Thiel, W.R. "A series of amide functionalized isoreticular metal organic frameworks." Microporous and Mesoporous Materials, 2014. 194:115-125. doi:10.1016/j.micromeso.2014.03.022

40. Gücüyener, C.; van den Bergh, J.; Gascon, J.; and Kapteijn, F. "Ethane/Ethene Separation Turned on Its Head: Selective Ethane Adsorption on the Metal-Organic Framework ZIF7 through a Gate-Opening Mechanism." Journal of the American Chemical Society, 2010. 132(50):17704-17706. doi:10.1021/ja1089765

41. Llewellyn, P.L; Bourrelly, S.; Vagner, C.; Heymans, N.; Leclerc, H.; Ghoufi, A.; Bazin, P. Vimont, A.; Daturi, M.; Devic, T.; Serre, C.; Weireld, G.D.; and Maurin, G. "Evaluation of MIL-47(V) for $\mathrm{CO}_{2}$-Related Applications." The Journal of Physical Chemistry C, 2013. 117(2):962-970. doi:10.1021/jp308525k

42. Mera, H.A.; Gomez-Ballesteros, J.L.; and Balbuena, P.B. "Structure and Dynamics of Carbon Dioxide, Nitrogen, Water, and Their Mixtures in Metal Organic Frameworks." Journal of Chemical \& Engineering Data, 2014. 59(10):2973-2981. doi:10.1021/je500100f 